\title{
Physical Medical Procedure
}

National Cancer Institute

\section{Source}

National Cancer Institute. Physical Medical Procedure. NCI Thesaurus. Code C98769.

A set of manual or instrumental activities that are intended to treat a health problem in order to achieve a positive result. 